Original Article

\title{
Structural, magnetic and dielectric properties of the novel magnetic spinel compounds $\mathrm{ZnCoSnO}_{4}$ and $\mathrm{ZnCoTiO}_{4}$
}

\author{
Domingo Ruiz-León $^{\mathrm{a}, * *}$, Federico Mompean ${ }^{\mathrm{b}}$, Jesús Prado-Gonjal ${ }^{\mathrm{b}}$, José F. Marco ${ }^{\mathrm{c}}$, \\ Mar García-Hernandez ${ }^{\mathrm{b}}$, Rainer Schmidt ${ }^{\mathrm{d}, \mathrm{e}, *}$

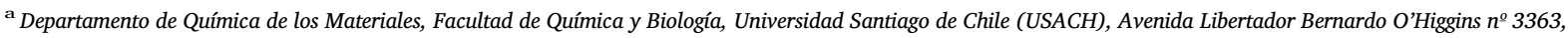 \\ Chile \\ ${ }^{\mathrm{b}}$ Instituto de Ciencia de Materiales de Madrid ICMM-CSIC, Sor Juana Inés de la Cruz 3, 28049 Madrid, Spain \\ ' Instituto de Química-Física Rocasolano, CSIC, 28006 Madrid, Spain \\ ' GFMC, Departamento de Física de Materiales, Facultad de Ciencias Físicas, Universidad Complutense de Madrid, 28040 Madrid, Spain \\ e Unidad Asociada "Laboratorio de heteroestructuras con aplicación en espintrónica", UCM/CSIC, 28049 Madrid, Spain
}

\section{A R T I C L E I N F O}

\section{Keywords:}

Transparent semiconductors

Ferrimagnetism

Ceramic

Synthesis

Dielectric

Properties

Spinel

\begin{abstract}
A B S T R A C T
The transparent semiconductor $\mathrm{Zn}_{2} \mathrm{SnO}_{4}$ with cubic spinel structure and the isostructural $\mathrm{Zn}_{2} \mathrm{TiO}_{4}$ have been magnetically doped with $\mathrm{Co}^{2+} . \mathrm{ZnCoSnO}_{4}$ and $\mathrm{ZnCoTiO}_{4}$ exhibit ferrimagnetism below $T_{\mathrm{N}} \approx 13 \mathrm{~K}$ and $T_{\mathrm{N}} \approx$ $17 \mathrm{~K}$. Ferrimagnetic moments are evident in $M$ vs $H$ curves below $T_{\mathrm{N}}$ by small hysteresis. Fits to strictly linear Curie-Weiss plots above $T_{\mathrm{N}}$ give $\mu_{\text {eff }} \approx 4.86 \mu_{\mathrm{B}}$ and $\approx 4.91 \mu_{\mathrm{B}}$ for $\mathrm{ZnCoSnO}_{4}$ and $\mathrm{ZnCoTiO}_{4}$, above theoretical predictions. Impedance spectroscopy data from sintered ceramic can be fitted with a standard equivalent circuit model based on two RC elements for bulk and GB areas. The relative dielectric permittivity of the bulk is $\approx 20$ and $\approx 30$ for $\mathrm{Zn}_{2} \mathrm{SnO}_{4}$ and $\mathrm{Zn}_{2} \mathrm{TiO}_{4}$. The semiconducting $\mathrm{ZnCoSnO}_{4}$ and $\mathrm{ZnCoTiO}_{4}$ ceramics exhibit bulk resistivity of $\approx 110^{6} \Omega \mathrm{cm}$ and $\approx 110^{5} \Omega \mathrm{cm}$ at $560 \mathrm{~K}\left(287^{\circ} \mathrm{C}\right)$, and bulk activation energies of $E_{\mathrm{A}} \approx 1.2 \mathrm{eV}$ and $1.1 \mathrm{eV}$.
\end{abstract}

\section{Introduction}

The spinel compound $\mathrm{Zn}_{2} \mathrm{SnO}_{4}$ is a prominent candidate for applications in dye-sensitized solar cells as a transparent semiconducting electrode [1-4], and in the decomposition of benzene and methylene as a photocatalyst [5-7]. For such applications $\mathrm{Zn}_{2} \mathrm{SnO}_{4}$ is considered a viable alternative to $\mathrm{TiO}_{2}$ based materials [8]. Furthermore, the use of spinel $\mathrm{Zn}_{2} \mathrm{SnO}_{4}$ in Li-ion batteries as an anode material has been demonstrated $[9,10]$. In the typical spinel structure the $\mathrm{O}^{2-}$ anions are cubic closed packed, whereas the cations occupy tetrahedral A-site interstitials by a fraction of $1 / 3$ and octahedral B-sites by $2 / 3$. In $\mathrm{Zn}_{2} \mathrm{SnO}_{4}$ the $\mathrm{Sn}^{4+}$ cations occupy octahedral sites and the $\mathrm{Zn}^{2+}$ tetrahedral and octahedral [] sites by equal amounts, i.e. $\mathrm{Zn}[\mathrm{ZnSn}] \mathrm{O}_{4}$.

In the work presented here, isovalent $\mathrm{Co}^{2+}$ magnetic doping of $\mathrm{Zn}_{2} \mathrm{SnO}_{4}$ and $\mathrm{Zn}_{2} \mathrm{TiO}_{4}$ by substitution of $\mathrm{Zn}^{2+}$ was studied in order to modify the optical absorption properties and increase the functionality of the material by adding a magnetic component to it. The end member spinel compounds $\mathrm{Co}_{2} \mathrm{SnO}_{4}$ and $\mathrm{Co}_{2} \mathrm{TiO}_{4}$ exhibit ferrimagnetic moments below $T_{\mathrm{N}}=41 \mathrm{~K}$ and $T_{\mathrm{N}}=48 \mathrm{~K}$ respectively $[11,12]$, which arise from the different magnetic moments of $\mathrm{Co}^{2+}$ on tetrahedral or octahedral sites. From an alternative point of view it may be regarded that the $\mathrm{Co}^{2+}$ magnetism in the ferrimagnetic spinels $\mathrm{Co}_{2} \mathrm{SnO}_{4}$ and $\mathrm{Co}_{2} \mathrm{TiO}_{4}$ was diluted by partial $\mathrm{Zn}^{2+}$ substitution.

Stoichiometric $\mathrm{ZnCoSnO}_{4}$ and $\mathrm{ZnCoTiO}_{4}$ were synthesized by conventional ceramic processing and the structural, microstructural, magnetic and dielectric properties were investigated. Powder X-ray diffraction (XRD) was employed to demonstrate that the $\mathrm{O}^{2-}$ sublattice of the spinel structure is ideally suited to accommodate $\mathrm{Sn}^{4+}, \mathrm{Zn}^{2+}$ and $\mathrm{Co}^{2+}$ cations. The valence state of Co was confirmed to be predominantly $2+$ in $\mathrm{ZnCoSnO}_{4}$ and $\mathrm{ZnCoTiO}_{4}$ from X-ray photoelectron spectroscopy (XPS) measurements, and the stoichiometry was checked using Energy Dispersive Spectroscopy (EDS).

Magnetic measurement data revealed strictly linear Curie-Weiss fits with $\mathrm{Co}^{2+}$ magnetic moments of $\mu_{\text {eff }} \approx 4.86 \mu_{\mathrm{B}}$ and $\approx 4.91 \mu_{\mathrm{B}}$ for $\mathrm{ZnCoSnO}_{4}$ and $\mathrm{ZnCoTiO}_{4}$, which is significantly above the maximum theoretical prediction of $4.19 \mu_{\mathrm{B}}$ associated with $\mathrm{Co}^{2+}$ high spin on octahedral sites. This unusually high $\mathrm{Co}^{2+}$ high spin moment may be explained by an exceptionally high orbit contribution or an unusual

\footnotetext{
* Corresponding author at: GFMC, Departamento de Física de Materiales, Facultad de Ciencias Físicas, Universidad Complutense de Madrid, 28040 Madrid, Spain.

** Corresponding author.

E-mail addresses: domingo.ruiz@usach.cl (D. Ruiz-León), rainerxschmidt@googlemail.com (R. Schmidt).
} 
anisotropy of the $g$-factors $g \|$ and $g_{\perp}$. Field cooled and zero field cooled magnetization $(M)$ vs temperature $(T)$ curves deviate from each other below $T_{\mathrm{N}}$, typical for a glassy magnetic spin behaviour.

Impedance spectroscopy measurements on $\mathrm{ZnCoSnO}_{4}$ and $\mathrm{ZnCoTiO}_{4}$ pressed and sintered pellets revealed insulating electronic conduction in both compounds. Intrinsic bulk and extrinsic grain boundary (GB) dielectric contributions were detected and analysed separately in terms of the resistivity and dielectric permittivity by fitting the dielectric data to a standard equivalent circuit model containing a series connection of two standard non-ideal RC elements or RQ elements, one each for bulk and GB contributions.

\section{Experimental}

$\mathrm{ZnCoSnO}_{4}$ and $\mathrm{ZnCoTiO}_{4}$ powders were synthesized using the conventional ceramic method of mixing and homogenizing the analytical grade $\mathrm{ZnO}$ (Merck, 99\%), $\mathrm{CoO}$ (Merck, 99.9\%) and $\mathrm{SnO}_{2}$ (Merck, 99.9\%) precursors in acetone using agate pestle and mortar. The mixed precursor powders were placed in an alumina crucible and calcined in a conventional Labtech LEF-115S muffle furnace for $24 \mathrm{~h}$ at two different temperatures of $900{ }^{\circ} \mathrm{C}$ and $1000{ }^{\circ} \mathrm{C}$ in two separate sample batches. After calcination, the samples were again ground in an agate mortar and calcined a second time at $900{ }^{\circ} \mathrm{C}$ or $1000^{\circ} \mathrm{C}$ for $12 \mathrm{~h}$ respectively to ensure complete homogeneity. For dielectric measurements the powders were pressed into pellets using a 1 ton uniaxial dye press and sintering of the green ceramic pellets was performed at $1000{ }^{\circ} \mathrm{C}$ and $1100{ }^{\circ} \mathrm{C}$ for the samples synthesized at $900{ }^{\circ} \mathrm{C}$ and $1000^{\circ} \mathrm{C}$ respectively, i.e. the sintering temperature was always $100^{\circ} \mathrm{C}$ above the synthesis to ensure optimum pellet compaction due to grain growth.

XRD pattern from the synthesized powders were collected at room temperature on a Siemens D5000 powder diffractometer with $\mathrm{CuK \alpha}$ radiation at diffraction angles in the range of $5^{\circ} \leq 2 \theta \leq 80^{\circ}$ with a counting time of $1 \mathrm{~s}$ over $0.02^{\circ}(2 \theta)$ steps. The analysis of XRD patterns was performed using the Crystal Impact Match program with the PDF-2 database. The lattice parameters were calculated using the LeBail method in the Fullprof software. XPS spectra were recorded with a CLAM2 electron spectrometer under vacuum below $1 \times 10^{-9} \mathrm{mbar}$, using $\mathrm{Mg}$ K $\alpha$ radiation (1253.6 eV) and a constant pass energy of $200 \mathrm{eV}$ and $20 \mathrm{eV}$ for the wide scan and narrow scan spectra, respectively. All binding energies ( $\mathrm{BE} \pm 0.2 \mathrm{eV}$ ) were charge-corrected to the $\mathrm{C} 1 \mathrm{~s}$ signal of the adventitious contamination carbon layer, set at $284.6 \mathrm{eV}$. The ceramic microstructure of sintered pellets was imaged using a JEOL JSM 6400 scanning electron microscope (SEM). The pellets had been polished, significantly thinned and thermally etched to obtain images representative of the bulk interior microstructure. Energy Dispersive Spectroscopy (EDS) was carried out for quantitative analysis of the stoichiometry using an INCAx-sight detector. The compositional EDS results were confirmed by Inductive-Coupled Plasma (ICP) spectroscopy using an ICP PERKIN ELMER mod. OPTIMA 2100 DV.

A Quantum Design SQUID MPMS was used to measure the powder magnetisation $M$ vs $T$ curves between $5 \mathrm{~K}$ and $320 \mathrm{~K}$ during heating under ZFC and FC conditions under an applied magnetic field of $H=100$ Oe. Furthermore, $M$ vs $H$ curves were measured at $5 \mathrm{~K}$ and $15 \mathrm{~K}$ below and above the ferrimagnetic transitions $T_{\mathrm{N}}$ to detect possible magnetic hysteresis.

The dielectric properties of the sintered pellets were measured by alternating current (AC) impedance spectroscopy using an Alpha Analyser Novocontrol system. Au electrodes were sputter deposited on both faces of the pellets and covered by Ag conductive paint. Prior to measurement the short sides of the pellets were polished thoroughly to avoid short-circuiting. Impedance measurements were performed at a frequency $(f)$ range of $1 \mathrm{~Hz}-10 \mathrm{MHz}$ with 6 measurement points per frequency decade using an applied AC voltage signal of $0.1 \mathrm{~V}$ amplitude under various $T$ between $160 \mathrm{~K}$ and $560 \mathrm{~K}$ upon heating. The dielectric data were collected in terms of the real and imaginary parts $\left(Z^{\prime}, Z^{\prime \prime}\right)$ of the complex impedance $Z^{*}=Z^{\prime}+\mathrm{i} Z^{\prime}$ " under steady state conditions,

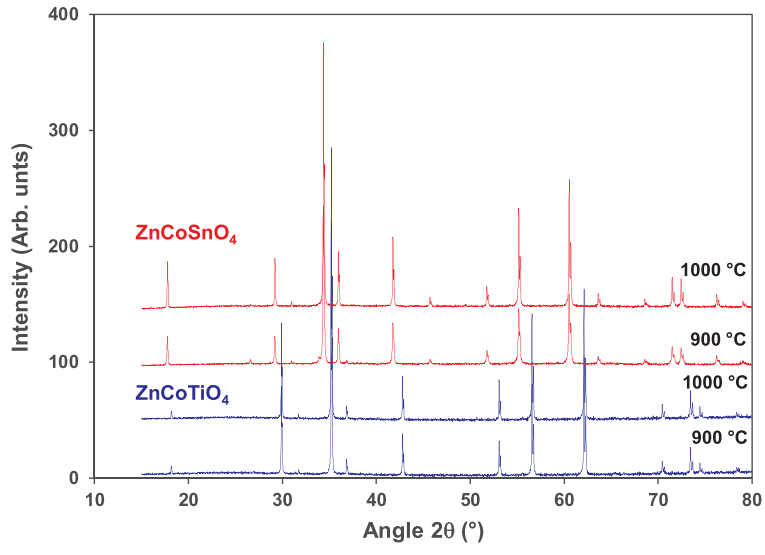

Fig. 1. Powder XRD pattern for spinel $\mathrm{ZnCoSnO}_{4}$ and $\mathrm{ZnCoTiO}_{4}$ heat treated at different temperatures. No secondary phases are evident. Large background signals from the fluorescence of Co were subtracted from the data.

where the selected $T$ was stabilised for $\approx 10 \mathrm{~min}$ before taking data. Equivalent circuit fitting of the dielectric data was performed by using commercial Z-View software.

\section{Results \& discussion}

\section{(A) Structural analysis}

Fig. 1 demonstrates that the XRD patterns of all synthesized $\mathrm{ZnCoSnO}_{4}$ and $\mathrm{ZnCoTiO}_{4}$ powders are strictly single phase and the crystal structure is a cubic spinel in the Fd3m space group. The unit cell parameters were calculated and are listed in Table 1 . Substitution of the $\mathrm{Zn}^{2+}$ cations with an ionic radius of $0.74 \AA$ (VI) in the octahedral or $0.60 \AA$ (IV) in the tetrahedral coordination, by $\mathrm{Co}^{2+}$ in the low spin (LS) or high spin (HS) configuration (VI: $0.65 \AA$ LS/0.75 $\mathrm{A} \mathrm{HS}$; IV: $0.58 \AA \mathrm{HS}$ ) [13] leads to isostructural phase pure compounds due to the good match of ionic radii.

Fig. 2a \& b shows the wide scan XPS spectra recorded from $\mathrm{ZnCoSnO}_{4}$ and $\mathrm{ZnCoTiO}_{4}$ samples. Only the more intense photoemission lines are labeled. The features of the Co $2 \mathrm{p}_{3 / 2}$ line were rather similar for all samples and Fig. $2 \mathrm{c}$ shows the $\mathrm{ZnCoTiO}_{4}$ Co $2 \mathrm{p}_{3 / 2}$ line as a representative example.

The main photoemission line is centered at $780.1 \mathrm{eV}$ and a strong shake-up satellite at 786. $2 \mathrm{eV}$ is observed. These spectral features and binding energy values are typical for $\mathrm{Co}^{2+}$ cations in spinel-related compounds [14]. The existence of some $\mathrm{Co}^{3+}$ cations was considered by including the characteristic photoemission line at $779.5 \mathrm{eV}$ into the fits. However, in all cases the quality of the fits did not improve as compared to only $\mathrm{Co}^{2+}$ cations and therefore, the presence of considerable amounts of $\mathrm{Co}^{3+}$ was discarded. The ceramic microstructure of the bulk interior areas of sintered pellets is demonstrated in Fig. 3, where a regular grain structure, and a narrow and uniform grain size distribution were evident in the secondary electron (SE) and backscattered (BE) SEM micrographs.

This indicates that the sintered ceramic pellets may be well-suited for dielectric spectroscopy measurements. The results from quantitative EDS analysis are summarized in Table 2 . It is indicated that all atomic percentages (at. \%) are approximately within the expected range

Table 1

Lattice parameters $\boldsymbol{a}$ calculated from powder XRD patterns for $\mathrm{ZnCoSnO}_{4}$ and $\mathrm{ZnCoTiO}_{4}$ heat treated at different temperatures.

\begin{tabular}{llllll}
\hline & $\mathrm{ZnCoSnO}_{4}$ & & & \multicolumn{2}{l}{$\mathrm{ZnCoTiO}_{4}$} \\
\cline { 5 - 6 } & & & & & \\
\cline { 5 - 6 }$(\AA)$ & $900{ }^{\circ} \mathrm{C}$ & $1000{ }^{\circ} \mathrm{C}$ & & $90{ }^{\circ} \mathrm{C}$ & $1000^{\circ} \mathrm{C}$ \\
& $8.6446(1)$ & $8.6465(1)$ & & $8.4457(1)$ & $8.4467(1)$
\end{tabular}



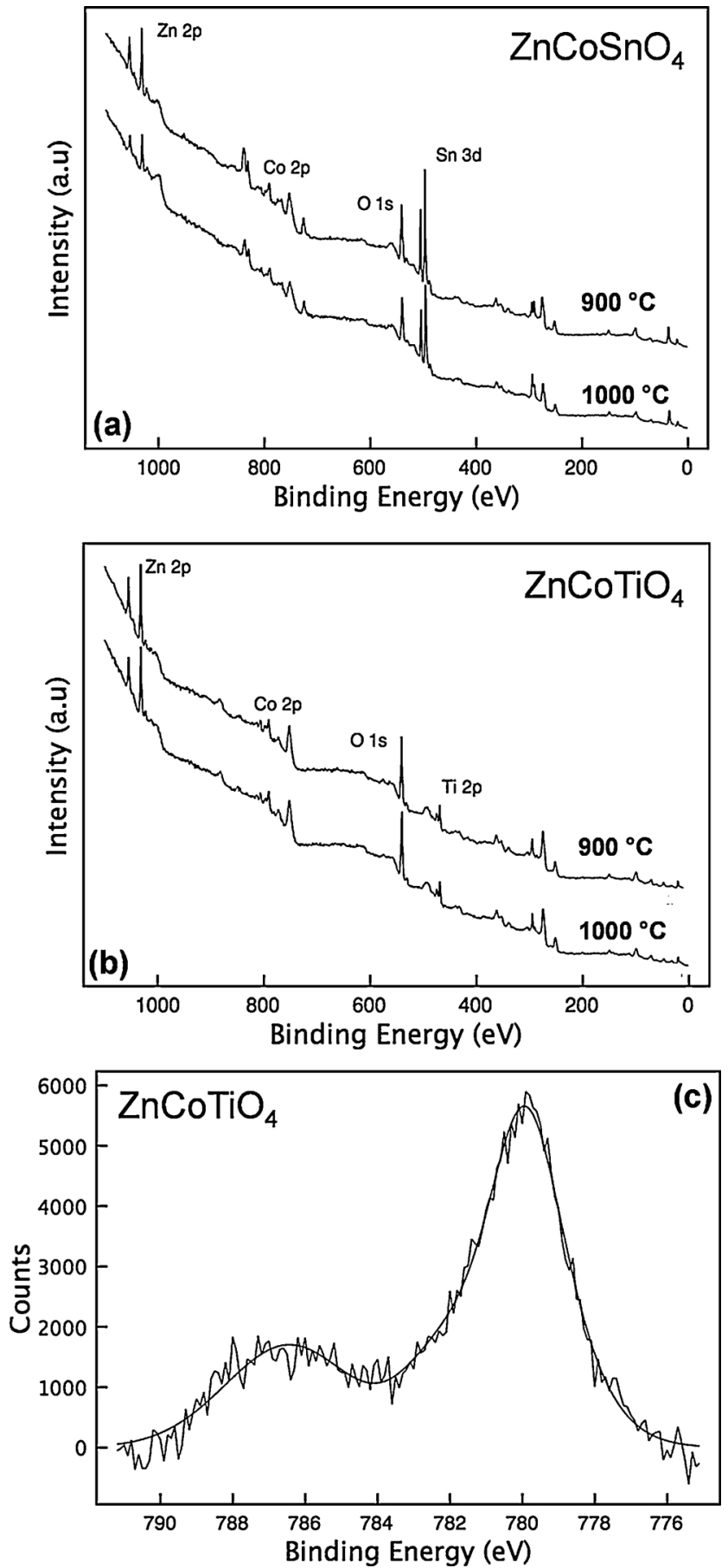

Fig. 2. Full XPS spectra for (a) $\mathrm{ZnCoSnO}_{4}$ and (b) $\mathrm{ZnCoTiO}_{4}$ heat treated at different temperatures. (c) Fits to the $2 \mathrm{p}$ Co peaks for $\mathrm{ZnCoTiO}_{4}$ treated at $1000{ }^{\circ} \mathrm{C}$. No $\mathrm{Co}^{3+}$ traces were detected.

considering the experimental error of EDS analysis of $\approx 2 \%$.

\section{(B) Magnetic properties}

The $M$ vs $T$ curves of $\mathrm{ZnCoSnO}_{4}$ and $\mathrm{ZnCoTiO}_{4}$ powder samples heat treated at $1000{ }^{\circ} \mathrm{C}$ (Fig. $4 \mathrm{a} \& \mathrm{~b}$ ) indicate $T_{\mathrm{N}} \approx 13$ and $T_{\mathrm{N}} \approx 17$ respectively. These values are considerably lower as compared to the isostructural compounds $\mathrm{Co}_{2} \mathrm{SnO}_{4}$ and $\mathrm{Co}_{2} \mathrm{TiO}_{4} \quad\left(T_{\mathrm{N}}=41 \mathrm{~K}\right.$ and $T_{\mathrm{N}}=48 \mathrm{~K}$ ), which may well be a result of the diluted $\mathrm{Co}^{2+}$ magnetism by $\mathrm{Zn}^{2+}$ substitution. Fig. 4 further indicates a separation of the ZFC and FC curves below $T_{\mathrm{N}}$, which is reminiscent of a glassy spin magnetic moment commonly observed in (anti)ferromagnetic and ferrimagnetic spinels [11,15-17]. The Fig. 4 insets demonstrate that the deviations between ZFC and FC curves (maxima in the ZFC curves) occur at temperatures very close to the Neel transitions $T_{\mathrm{N}}$ as indicated by the maxima in the differentiated FC curves, for both $\mathrm{ZnCoSnO}_{4}$ and $\mathrm{ZnCoTiO}_{4}$. Therefore, both compounds may enter into a glassy spin state just below $T_{\mathrm{N}}$, which had been observed previously in $\mathrm{Co}_{2} \mathrm{SnO}_{4}$ $[11,18,19]$.

The magnetic data from $\mathrm{ZnCoSnO}_{4}$ and $\mathrm{ZnCoTiO}_{4}$ powders heat treated at $1100{ }^{\circ} \mathrm{C}$ show the equivalent $T_{\mathrm{N}}$ transition temperatures, glassy spin behaviour and ferrimagnetism (data not shown). Therefore, it can be concluded that the magnetism in $\mathrm{ZnCoSnO}_{4}$ and $\mathrm{ZnCoTiO}_{4}$ powders is not affected qualitatively by the differences in heat treatment. The ferrimagnetic moments were confirmed from the hysteretic $M$ vs $H$ curves at $T=4.2 \mathrm{~K}$ below $T_{\mathrm{N}}$, whereas above $T_{\mathrm{N}}$ at $20 \mathrm{~K}$ and $40 \mathrm{~K}$ approximately linear behaviour is indicated in Fig. $5 \mathrm{a} \& \mathrm{~b}$. The ferrimagnetic moment arises from the different magnetic moments of the $\mathrm{Co}^{2+}$ cations situated at tetrahedral or octahedral sites, since the octahedral coordination entails orbit contributions, which are absent in the tetrahedral coordination [20]. This ferrimagnetic moment detected here had been demonstrated previously in the end member compounds $\mathrm{Co}_{2} \mathrm{SnO}_{4}$ and $\mathrm{Co}_{2} \mathrm{TiO}_{4}[11,12]$.

The magnetic behaviour of both $\mathrm{ZnCoSnO}_{4}$ and $\mathrm{ZnCoTiO}_{4}$ above $T_{\mathrm{N}}$ in the paramagnetic regime is dominated by strictly linear susceptibility $1 / \chi$ vs $T$ Curie-Weiss plots, which indicate a conventional paramagnetism of non-interacting magnetic spin moments. However, the linear fits to the Curie-Weiss plots and calculating the total effective magnetic moment $\mu_{\text {eff }}$ leads to values in the range of $\approx 4.9 \mu_{\mathrm{B}}$ that are significantly above the maximum values predicted. This is the case even by assuming an HS scenario and full octahedral occupation of $\mathrm{Co}^{2+}$ cations with the higher predicted HS moment of $4.16 \mu_{\mathrm{B}}$ as compared to the predicted tetrahedral HS moment of $3.88 \mu_{\mathrm{B}}$. All $\mu_{\text {eff }}$ values calculated from the strictly linear Curie-Weiss plots shown in Fig. 6 are summarized in Table 3. The theoretical predictions for the total $\mu_{\text {eff }}$ values were calculated assuming a spin-only moment of the tetrahedral $\mathrm{Co}^{2+}$ cations, but both spin and orbit contributions for the octahedral $\mathrm{Co}^{2+}$ cations in the HS state. The possibility of a mixed LS/IS/HS spinstate scenario on octahedral $\mathrm{Co}^{3+}$ lattice sites has been proposed previously in $\mathrm{LaCoO}_{3}$ perovskites (here in the $\mathrm{Co}^{2+}$ spinel only LS/HS mixing on octahedral sites would be possible) [21-26], but this scenario was discarded in this study due to the high $\mu_{\text {eff }}$ values encountered.

The detection of an increased magnetic moment in $\mathrm{Co}^{2+}$ magnetic cations may be interpreted in terms of an unusually high orbit contribution and strong spin-orbit coupling. However, the detected moment of $\mu_{\text {eff }} \approx 4.9 \mu_{\mathrm{B}}$ is considerably higher than the predicted $4.16 \mu_{\mathrm{B}}$ for a strictly octahedral occupation of $\mathrm{Co}^{2+}$, which in fact may be unrealistic. Due to a close match of the ionic radii on octahedral $\left(\mathrm{Zn}^{2+}\right.$ :

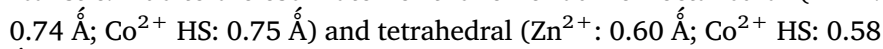
$\AA$ Á) sites, a good dispersion of $\mathrm{Zn}^{2+}$ and $\mathrm{Co}^{2+}$ on tetrahedral and octahedral sites is likely, which would imply an inversion parameter of the spinel structure near $\nu \approx 0.5$. In this case the detected total $\mu_{\text {eff }}$ would be $\approx 20 \%$ above theoretical predictions. It may be doubtful if this large increase can be explained by unusual orbit contributions only, and the alternative explanation of an unusual anisotropy of the $g$-factors $g \|$ and $g_{\perp}$ may be considered as well [20]. A possible $\mathrm{Co}^{2+}$ excess in the samples could be discarded from the EDS results presented in Table 2. Furthermore, ICP spectroscopy data did not give any indications for $\mathrm{Co}^{2+}$ excess either.

\section{(C) Dielectric properties}

Impedance spectroscopy data from $\mathrm{ZnCoSnO}_{4}$ and $\mathrm{ZnCoTiO}_{4}$ ceramic pellets sintered at $1000{ }^{\circ} \mathrm{C}$ and $1100{ }^{\circ} \mathrm{C}$ are presented in Fig. 7 in form of the complex impedance plots of $Z$ " vs $Z$. The characteristic semicircles indicate different dielectric contributions, where the data 

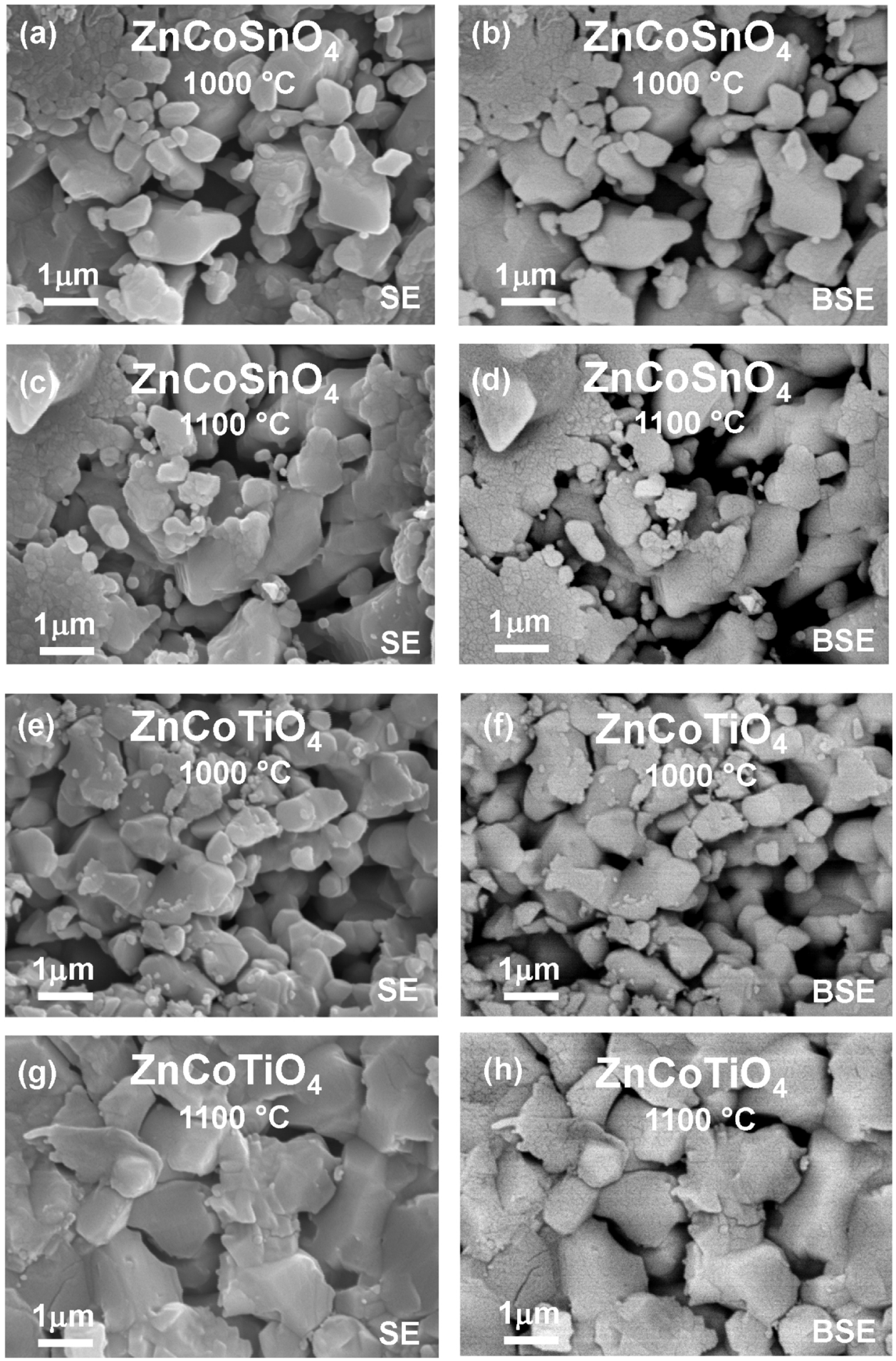

Fig. 3. Secondary electron (SE) and backscattered electron (BSE) SEM micrographs for $\mathrm{ZnCoSnO}_{4}$ (a-d) and $\mathrm{ZnCoTiO}_{4}(\mathbf{e}-\mathbf{h})$ ceramic pellets sintered at $1000{ }^{\circ} \mathrm{C}$ and $1100^{\circ} \mathrm{C}$ as indicated.

Table 2

Cation ratios in atomic percentage from quantitative analysis by EDS for $\mathrm{ZnCoSnO}_{4}$ and $\mathrm{ZnCoTiO}_{4}$ heat treated at different temperatures. Uncertainties are in the range of $\approx 2 \%$

\begin{tabular}{|c|c|c|c|c|}
\hline \multirow[b]{2}{*}{ Synthesis Temperature } & \multicolumn{2}{|c|}{$\mathrm{ZnCoSnO}_{4}$} & \multicolumn{2}{|c|}{$\mathrm{ZnCoTiO}_{4}$} \\
\hline & $900{ }^{\circ} \mathrm{C}$ & $1000^{\circ} \mathrm{C}$ & $900{ }^{\circ} \mathrm{C}$ & $1000^{\circ} \mathrm{C}$ \\
\hline Zn (at. \%) & $31.6(6)$ & $32.5(7)$ & $33.0(7)$ & $32.6(7)$ \\
\hline Co (at. \%) & $32.8(7)$ & $32.4(6)$ & $32.8(7)$ & $33.6(7)$ \\
\hline Sn/Ti (at. \%) & $35.6(7)$ & $35.1(7)$ & $34.2(7)$ & $33.8(7)$ \\
\hline
\end{tabular}

from $1100{ }^{\circ} \mathrm{C}$ treated $\mathrm{ZnCoSnO}_{4}$ ceramics (Fig. $7 \mathrm{~b}$ ) shows the signs of two strongly overlapping semicircles, whereas the data from the $1100{ }^{\circ} \mathrm{C}$ sample indicate electronic homogeneity. On the other hand, the $\mathrm{ZnCoTiO}_{4}$ ceramics sintered at $1000^{\circ} \mathrm{C}$ and $1100{ }^{\circ} \mathrm{C}$ both show the clear signs of two dielectric contributions (Fig. 7c \& d), which can be clearly assigned to intrinsic bulk and grain boundary (GB) [27,28].

It is interesting to note that increasing the sintering temperature in $\mathrm{ZnCoSnO}_{4}$ ceramics does not improve the sample's homogeneity but leads to the formation of a dielectrically distinct GB contribution. This 

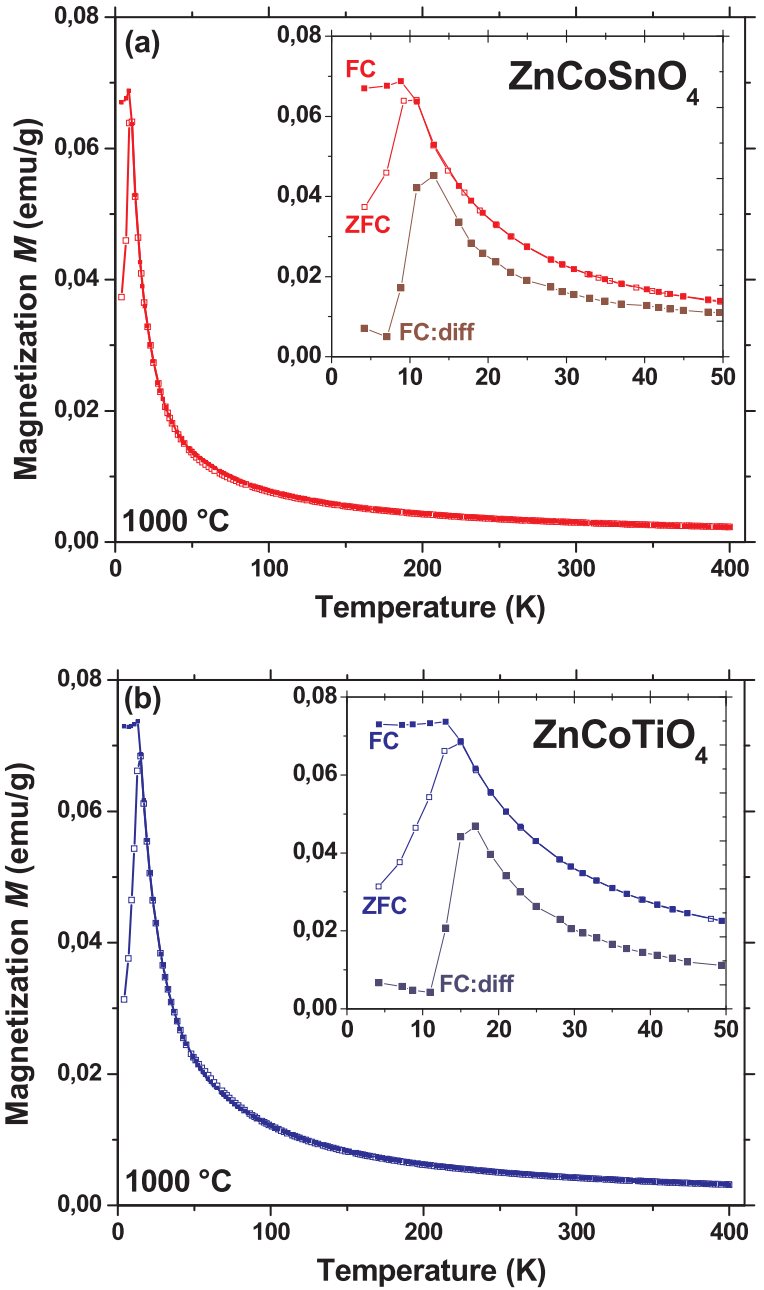

Fig. 4. Magnetization $M(\mathrm{emu} / \mathrm{g}$ ) vs $T(\mathrm{~K})$ plots collected under ZFC (open symbols) and FC (filled symbols) conditions for (a) $\mathrm{ZnCoSnO}_{4}$ (red symbols) and (b) $\mathrm{ZnCoTiO}_{4}$ (blue symbols) powders heat treated at $1000{ }^{\circ} \mathrm{C}$. The figure insets show low $T$ magnifications and the differentiated FC curves $\mathrm{d} M / \mathrm{d} T$ vs $T$ (brown and dark blue symbols). The maxima in the $\mathrm{d} M / \mathrm{d} T$ vs $T$ curves were regarded the transition temperatures $T_{\mathrm{N}}$, whereas the peaks in the ZFC curves indicate glassy spin behaviour below $T_{\mathrm{N}}$. (For interpretation of the references to colour in this figure legend, the reader is referred to the web version of this article).

points towards a certain degree of chemical inhomogeneity, which may arise within the $\mathrm{ZnCoSnO}_{4}$ ceramics at higher sintering temperatures when the diffusivity of the constituting atoms increases. Although this behaviour may be reminiscent of the formation of GB barrier layers in dielectric ceramics $[29,30]$, the diameter of the GB semicircle in the $1100{ }^{\circ} \mathrm{C}$ sintered $\mathrm{ZnCoSnO}_{4}$ ceramic is smaller as compared to the bulk and thus, the barrier effect may not be relevant for potential applications. The dielectric data presented in Fig. 7 were fitted to a standard equivalent circuit model containing RC elements of ideal resistors and parallel non-ideal capacitors represented by Constant-Phase element (CPEs), i.e. R-CPE or RQ elements. In the case of two R-CPE elements, they were connected in series to obtain a valid fit and the resistance and capacitance of bulk and GB contributions were extracted [31]. Fig. 7 indicates a good fit, which was reflected in low fitting errors $(<5 \%)$. The bulk and GB resistance and capacitance values extracted from the fits were normalized by the pellet geometry and plotted in form of the electrical resistivity $\rho$ vs $1 / T$ (Fig. 8) and the relative dielectric permittivity $\varepsilon$ vs T (Fig. 9), both on semi-logarithmic axes. The charge transport activation energies $E_{\mathrm{A}}(\mathrm{eV})$, the $\varepsilon$ values and the resistivity obtained at $560 \mathrm{~K}$ are summarized in Table 4 . The resistivity $\rho$
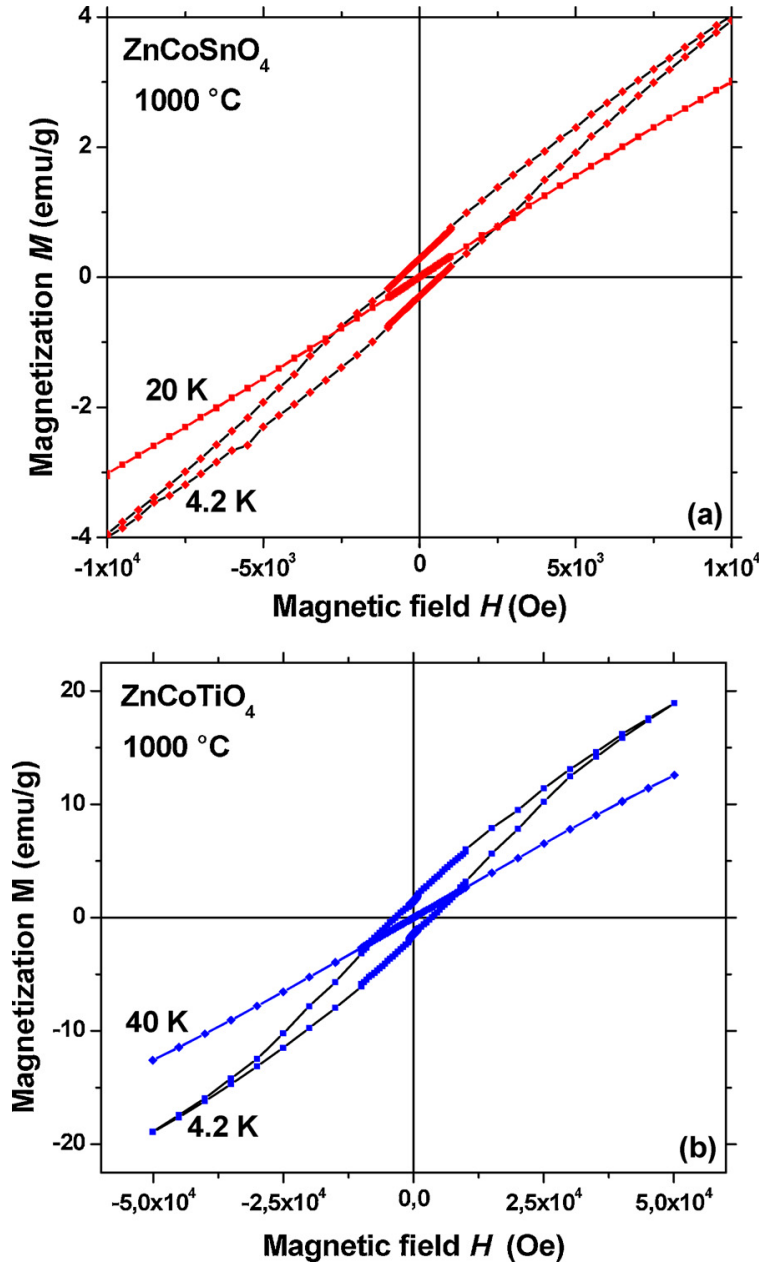

Fig. 5. Magnetization $M$ (emu/g) vs applied magnetic field $H(\mathrm{Oe})$ plots for (a) $\mathrm{ZnCoSnO}_{4}$ and (b) $\mathrm{ZnCoTiO}_{4}$ powders heat treated at $1000{ }^{\circ} \mathrm{C}$. Small hysteresis loops for $T<T_{\mathrm{N}}$ indicate net ferrimagnetic moments.

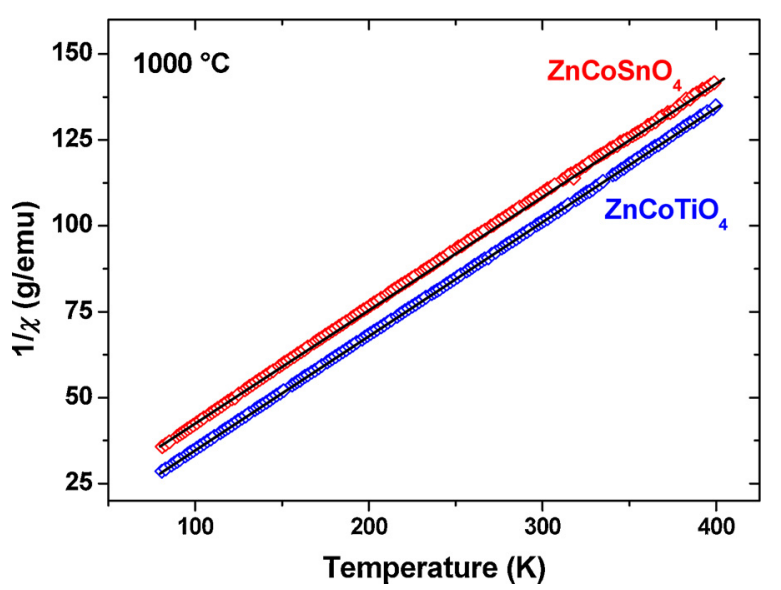

Fig. 6. Curie-Weiss plots of $1 / \chi$ vs $T$ for $\mathrm{ZnCoSnO}_{4}$ and $\mathrm{ZnCoTiO}_{4}$ powders heat treated at $1000^{\circ} \mathrm{C}$. Both curves show excellent linearity.

Table 3

Total efficient magnetic moment $\mu_{\text {eff }}$ for $\mathrm{ZnCoSnO}_{4}$ and $\mathrm{ZnCoTiO}_{4}$ heat treated at different temperatures. Uncertainties are below $1 \%$.

\begin{tabular}{|c|c|c|c|c|}
\hline \multirow{3}{*}{$\begin{array}{l}\text { Synthesis Temperature } \\
\mu_{\text {eff }}\left(\mu_{\mathrm{B}}\right)\end{array}$} & \multicolumn{2}{|c|}{$\mathrm{ZnCoSnO}_{4}$} & \multicolumn{2}{|c|}{$\mathrm{ZnCoTiO}_{4}$} \\
\hline & $900^{\circ} \mathrm{C}$ & $1000^{\circ} \mathrm{C}$ & $900^{\circ} \mathrm{C}$ & $1000^{\circ} \mathrm{C}$ \\
\hline & 4.91(1) & 4.91(1) & $4.73(1)$ & $4.91(1)$ \\
\hline
\end{tabular}



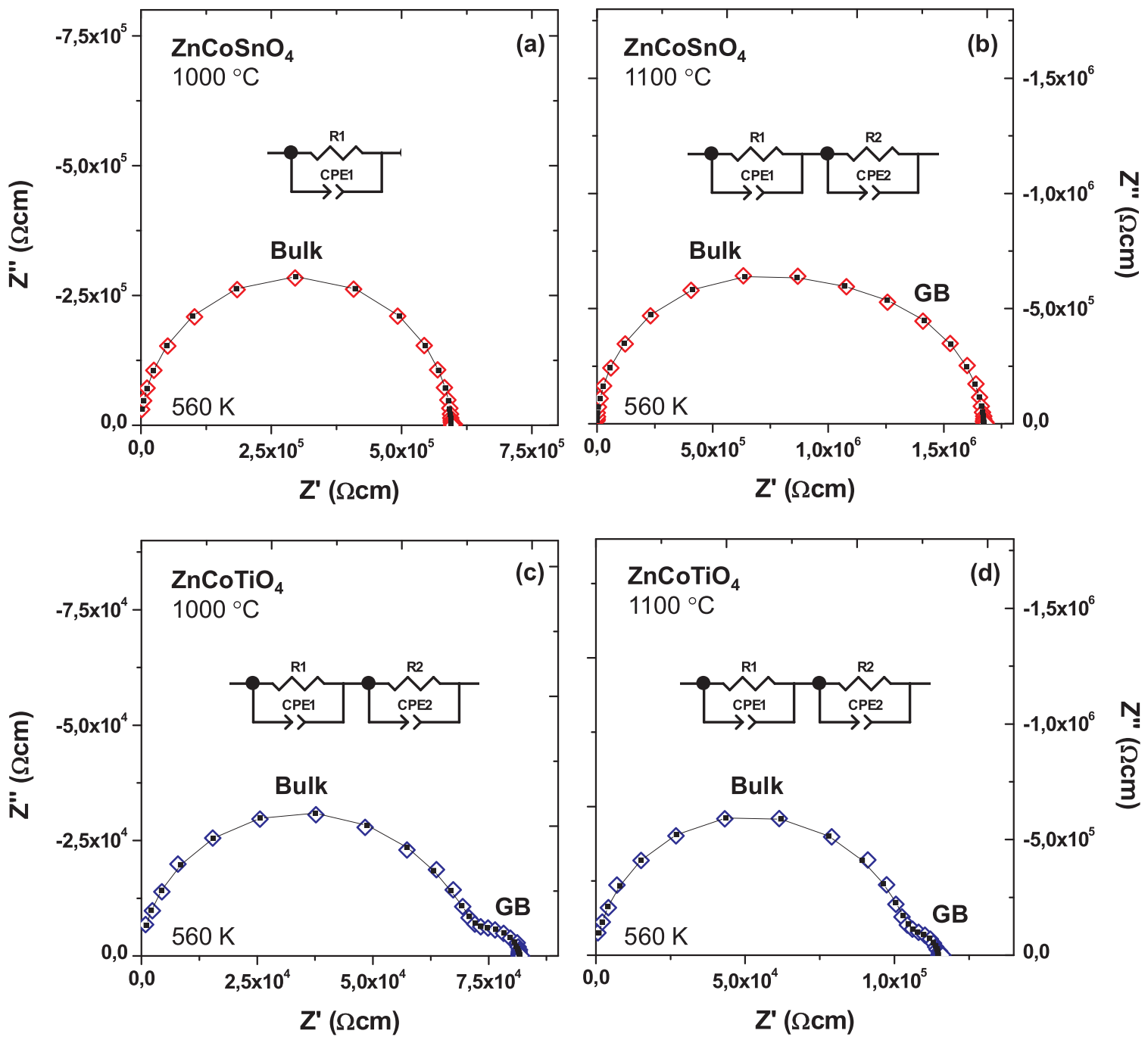

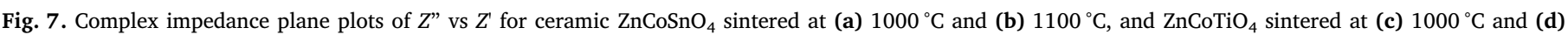

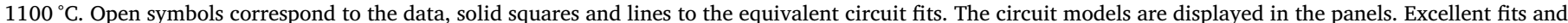
bulk and GB dielectric contributions are evident.

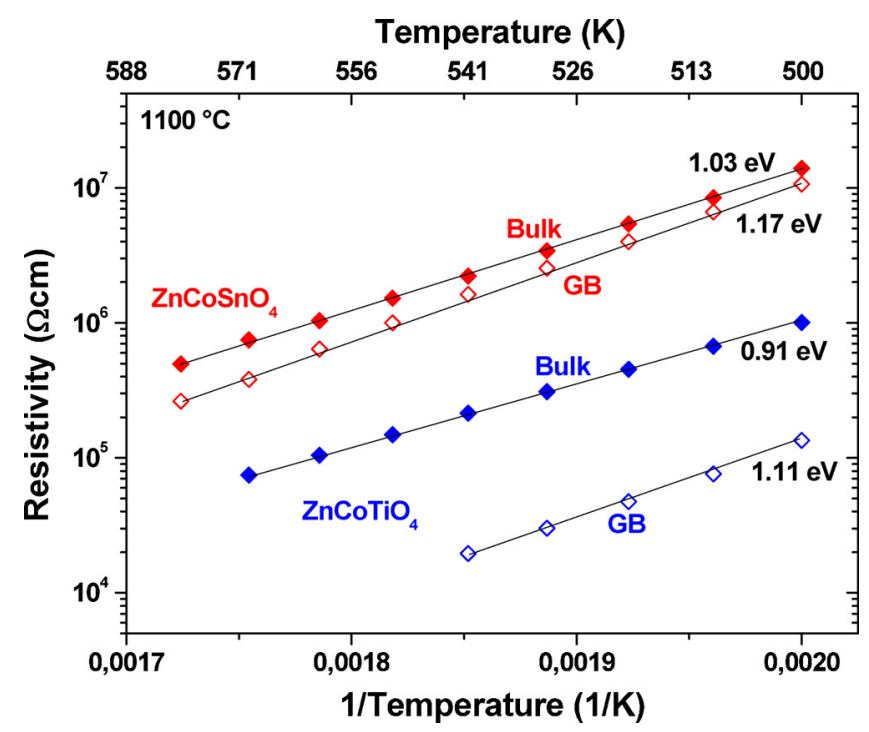

Fig. 8. Resistivity vs $1 / T$ for bulk and GB dielectric contributions obtained from equivalent circuit fits for ceramic $\mathrm{ZnCoSnO}_{4}$ and $\mathrm{ZnCoTiO}_{4}$ sintered at $1100{ }^{\circ} \mathrm{C}$. Good linearity indicates thermally activated electron transport. The displayed activation energies $E_{\mathrm{A}}(\mathrm{eV})$ were obtained from the respective Arrhenius plots.

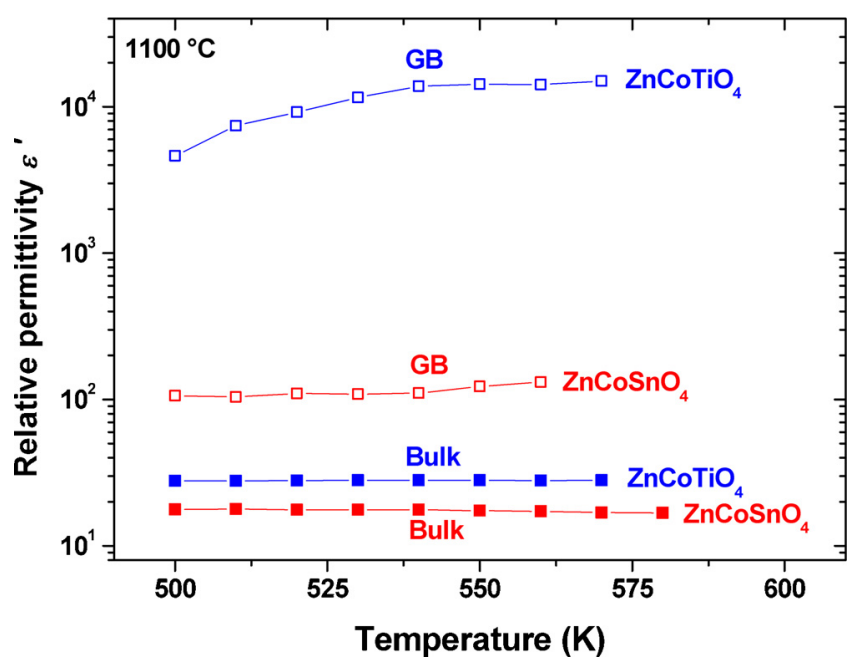

Fig. 9. Relative dielectric permittivity vs $T$ for bulk and GB dielectric contributions obtained from equivalent circuit fits for ceramic $\mathrm{ZnCoSnO}_{4}$ and $\mathrm{ZnCoTiO}_{4}$ sintered at $1100{ }^{\circ} \mathrm{C}$. 
Table 4

Bulk and GB resistivity $\rho$, activation energy $E_{\mathrm{A}}$ and dielectric permittivity $\varepsilon$ for $\mathrm{ZnCoSnO}_{4}$ and $\mathrm{ZnCoTiO}_{4}$ ceramics sintered at different temperatures.

\begin{tabular}{|c|c|c|c|c|}
\hline & $\mathrm{ZnCoSnO}_{4}$ & & $\mathrm{ZnCoTiO}_{4}$ & \\
\hline Sintering Temperature & $1000^{\circ} \mathrm{C}$ & $1100{ }^{\circ} \mathrm{C}$ & $1000^{\circ} \mathrm{C}$ & $1100^{\circ} \mathrm{C}$ \\
\hline$\rho(560 \mathrm{~K})$ Bulk $(\Omega \mathrm{cm})$ & $5.9610^{5} \pm 410^{2}$ & $1.0410^{6} \pm 310^{4}$ & $7.0510^{4} \pm 310^{2}$ & $1.0410^{5} \pm 410^{2}$ \\
\hline$\rho(560 \mathrm{~K}) \mathrm{GB}(\Omega \mathrm{cm})$ & - & $6.3910^{5} \pm 310^{4}$ & $1.1110^{4} \pm 310^{2}$ & $1.0510^{4} \pm 410^{2}$ \\
\hline$E_{\mathrm{A}}$ Bulk $(\mathrm{eV})$ & $1.085(1)$ & $1.032(8)$ & $0.915(5)$ & $0.915(3)$ \\
\hline$E_{\mathrm{A}} \mathrm{GB}(\mathrm{eV})$ & - & $1.17(2)$ & $1.04(2)$ & $1.11(2)$ \\
\hline$\varepsilon$ Bulk & $22.8(1)$ & $17.4(4)$ & $31.3(1)$ & $28.0(1)$ \\
\hline$\varepsilon \mathrm{GB}$ & - & $\approx 120 \pm 20$ & $\approx 10,000 \pm 2000$ & $\approx 10,000 \pm 4000$ \\
\hline
\end{tabular}

vs $1 / T$ plots in Fig. 8 show good linearity for $\mathrm{ZnCoSnO}_{4}$ and $\mathrm{ZnCoTiO}_{4}$ ceramics sintered at $1100{ }^{\circ} \mathrm{C}$, which indicates a conventional thermally activated electron charge transport. The $E_{\mathrm{A}}$ values are indicated in Fig. 8 and summarized in Table 4.

All $E_{\mathrm{A}}$ values are relatively large for electronic charge transport, but none of the typical signs of ionic charge transport were detected and the charge carriers may well be electrons. The $E_{\mathrm{A}}$ values for the GB contributions are slightly higher, which indicates a lower concentration of charge carriers within the GB areas. Nevertheless, the nominal values of the GB resistance or resistivity are smaller as compared to the bulk values, which is evidenced in Figs. 7 and 8 by the size of the respective semicircles and the resistance or resistivity values extracted. This can be understood by a geometrical effect of reduced thickness of the GB areas as compared to the bulk leading to lower resistance, whereas the effective resistivity within the GB areas may in fact be larger than in the bulk.

All data in Figs. 7 \& Figure 8 were normalized to the same macroscopic pellet geometry to obtain the resistivity, which may not be adequate for the microscopically thin GB areas and the effective resistivity within GB areas may thus be significantly higher than the bulk, i.e. charge transport can be assumed to be hindered by the GBs. Fig. 9 indicates an intrinsic bulk $\varepsilon \approx 20$ and $\approx 30$ for $\mathrm{ZnCoSnO}_{4}$ and $\mathrm{ZnCoTiO}_{4}$ ceramics sintered at $1100{ }^{\circ} \mathrm{C}$, respectively.

The relative dielectric permittivity values for the $1000^{\circ} \mathrm{C}$ sintered pellets are marginally higher in both cases. All $\varepsilon$ values are relatively low, especially for $\mathrm{ZnCoTiO}_{4}$. Quite often, $\mathrm{Ti}^{4+}$ on octahedral sites leads to larger permittivity values in complex oxides, e.g. $\approx 100$ in $\mathrm{CaCu}_{3} \mathrm{Ti}_{4} \mathrm{O}_{12}$ [29] and $\mathrm{TiO}_{2}$ [32], or even to ferroelectricity, e.g. in $\mathrm{BaTiO}_{3}$ [33] and $\mathrm{PbTiO}_{3}$ [34]. It can be concluded that $\mathrm{ZnCoSnO}_{4}$ and $\mathrm{ZnCoTiO}_{4}$ exhibit a dielectric permittivity that is typical for standard dielectric materials. The GB permittivity is higher than the bulk permittivity by a factor of $\approx 5-10$ and $\approx 300$ for $\mathrm{ZnCoSnO}_{4}$ and $\mathrm{ZnCoTiO}_{4}$ ceramics, respectively. Both values are in a typical range for extrinsic GB contributions [35].

\section{Conclusions}

The compounds $\mathrm{ZnCoSnO}_{4}$ and $\mathrm{ZnCoTiO}_{4}$ have been synthesized successfully and characterized comprehensively. Both compounds crystallize in a cubic spinel structure and exhibit ferrimagnetism below $T_{\mathrm{N}}$. The $\mathrm{Co}^{2+}$ spin states were suggested to be HS in both compounds above theoretical predictions. Electronic conduction was detected at elevated temperature and $\mathrm{ZnCoSnO}_{4}$ and $\mathrm{ZnCoTiO}_{4}$ may be regarded bad electronic insulators or high resistivity semiconductors. This work demonstrates that transparent semiconductors like $\mathrm{Zn}_{2} \mathrm{SnO}_{4}$ can be doped magnetically to increase their functionality.

\section{Acknowledgments}

R.S. acknowledges a travel grant (Convenio Internacional) from the Universidad Complutense de Madrid to visit the USACH in Chile. JPG thanks the Spanish MINECO for granting a Juan de la Cierva fellowship. Spanish MINECO grant MAT2014-52405-C2-2-R is also acknowledged.

\section{References}

[1] T. Lana-Villarreal, G. Boschloo, A. Hagfeldt, Nanostructured zinc stannate as semiconductor working electrodes for dye-sensitized solar cells, J. Phys. Chem. C 111 (14) (2007) 5549.

[2] B. Tan, E. Toman, Y. Li, Y. Wu, Zinc stannate $\left(\mathrm{Zn}_{2} \mathrm{SnO}_{4}\right)$ dye-sensitized solar cells, J. Am. Chem. Soc. 129 (14) (2007) 4162.

[3] S.S. Shin, W.S. Yang, J.H. Noh, J.H. Suk, N.J. Jeon, J.H. Park, J.S. Kim, W.M. Seong, S.I. Seok, High-performance flexible perovskite solar cells exploiting $\mathrm{Zn}_{2} \mathrm{SnO}_{4}$ prepared in solution below $100{ }^{\circ} \mathrm{C}$, Nat. Commun. 6 (2015) 7410.

[4] Z. Li, Y. Zhou, J. Zhang, W. Tu, Q. Liu, T. Yu, Z. Zou, Hexagonal nanoplate-textured micro-octahedron $\mathrm{Zn}_{2} \mathrm{SnO}_{4}$ : combined effects toward enhanced efficiencies of dyesensitized solar cell and photoreduction of $\mathrm{CO} 2$ into hydrocarbon fuels, Cryst. Growth Des. 12 (3) (2012) 1476.

[5] M.A. Alpuche-Aviles, Y. Wu, Photoelectrochemical study of the band structure of $\mathrm{Zn}_{2} \mathrm{SnO}_{4}$ prepared by the hydrothermal method, J. Am. Chem. Soc. 131 (9) (2009) 3216.

[6] C.K. Govindappa, V.T. Venkatarangaiah, S.B.A. Hamid, Electrochemical generation of cubic shaped nano $\mathrm{Zn}_{2} \mathrm{SnO}_{4}$ photocatalysts, Nano-Micro Lett. 5 (2) (2013) 101.

[7] W. Cun, W. Xinming, Z. Jincai, M. Bixian, S. Guoying, P. Ping'an, F. Jiamo, Synthesis, characterization and photocatalytic property of nano-sized $\mathrm{Zn}_{2} \mathrm{SnO}_{4}, \mathrm{~J}$. Mater. Sci. 37 (14) (2002) 2989.

[8] D. Ruiz-León, R.E. Avila, C.J. Venegas, Synthesis and electrical characterization of zinc stannate (zto) prepared by hydrothermal method, J. Chil. Chem. Soc. 60 (3) (2015) 3029.

[9] X.J. Zhu, L.M. Geng, F.Q. Zhang, Y.X. Liu, L.B. Cheng, Synthesis and performance of $\mathrm{Zn}_{2} \mathrm{SnO}_{4}$ as anode materials for lithium ion batteries by hydrothermal method, J. Power Sources 189 (1) (2009) 828.

[10] W.S. Yuan, Y.W. Tian, G.Q. Liu, Synthesis and electrochemical properties of pure phase $\mathrm{Zn}_{2} \mathrm{SnO}_{4}$ and composite $\mathrm{Zn}_{2} \mathrm{SnO}_{4} / \mathrm{C}$, J. Alloys Compd. 506 (2) (2010) 683.

[11] S. Nayak, K. Dasari, D.C. Joshi, P. Pramanik, R. Palai, A. Waske, R.N. Chauhan, N. Tiwari, T. Sarkar, S. Thota, Low-temperature anomalous magnetic behavior of $\mathrm{Co}_{2} \mathrm{TiO}_{4}$ and $\mathrm{Co}_{2} \mathrm{SnO}_{4}$, J. Appl. Phys. 120 (16) (2016) 163905.

[12] S. Nayak, S. Thota, D.C. Joshi, M. Krautz, A. Waske, A. Behler, J. Eckert, T. Sarkar, M.S. Andersson, R. Mathieu, et al., Magnetic compensation, field-dependent magnetization reversal, and complex magnetic ordering in $\mathrm{CoTiO}_{4}$, Phys. Rev. B 92 (21) (2015) 214434

[13] R.D. Shannon, Revised effective ionic radii and systematic studies of interatomic distances in Halides and chalcogenides, Acta Crystallogr. A 32 (1976) 751.

[14] J.F. Marco, J.R. Gancedo, J. Ortiz, J.L. Gautier, Characterization of the spinel-related oxides $\mathrm{Ni}_{\mathrm{x}} \mathrm{Co}_{3-\mathrm{x}} \mathrm{O}_{4}(\mathrm{x}=0.3,1.3,1.8)$ prepared by spray pyrolysis at $350{ }^{\circ} \mathrm{C}$, Appl. Surf. Sci. 227 (1) (2004) 175.

[15] D. Fiorani, S. Viticoli, J.L. Dormann, J.L. Tholence, A.P. Murani, Spin-glass behavior in an antiferromagnetic frustrated spinel: $\mathrm{ZnCr}_{1.6} \mathrm{Ga}_{0.4} \mathrm{O}_{4}$, Phys. Rev. B 30 (5) (1984) 2776 .

[16] Y.I. Jang, F.C. Chou, Y.M. Chiang, Spin-glass behavior in $\mathrm{LiMn}_{2} \mathrm{O}_{4}$ spinel, Appl. Phys. Lett. 74 (17) (1999) 2504.

[17] C.P. Poole, H.A. Farach, Magnetic phase diagram of spinel spin-glasses, Zeitschrift für Physik Phys. B Condens. Matter 47 (1) (1982) 55.

[18] S. Thota, V. Narang, S. Nayak, S. Sambasivam, B.C. Choi, T. Sarkar, M.S. Andersson, R. Mathieu, M.S. Seehra, On the nature of magnetic state in the spinel $\mathrm{Co}_{2} \mathrm{SnO}_{4}, \mathrm{~J}$. Phys. Condens. Matter 27 (16) (2015) 166001.

[19] S. Thota, M.S. Seehra, Co-existence of ferrimagnetism and spin-glass state in the spinel $\mathrm{Co}_{2} \mathrm{SnO}_{4}$, J. Appl. Phys. 113 (20) (2013) 203905.

[20] R.L. Carlin, A.J. van Duyneveldt, Magnetic Properties of Transition Metal Compounds, Springer-Verlag, New York Heidelberg Berlin, 1977.

[21] D.P. Kozlenko, N.O. Golosova, Z. Jirak, L.S. Dubrovinsky, B.N. Savenko, M.G. Tucker, Y.L. Godec, V.P. Glazkov, Temperature- and pressure-driven spin-state transitions in $\mathrm{LaCoO}_{3}$, Phys. Rev. B 75 (6) (2007) 064422.

[22] R. Schmidt, J. Wu, C. Leighton, I. Terry, Dielectric response to the low-temperature magnetic defect structure and spin state transition in polycrystalline $\mathrm{LaCoO}_{3}$, Phys. Rev. B 79 (2009) 125105.

[23] J. Prado-Gonjal, J. Gutiérrez-Seijas, I.H. Ansorregui, E. Morán, I. Terry, R. Schmidt, The role of defects in microwave and conventionally synthesized LaCoO3 perovskite, J. Eur. Ceram. Soc. 36 (5) (2016) 1197.

[24] E.L. Nagaev, A.I. Podelshchikov, Phase separation and resistivity jumps in Co compounds and other materials with low-spin-high-spin transitions, J. Phys.Condens. Matter 8 (30) (1996) 5611.

[25] J. Gutiérrez Seijas, J. Prado-Gonjal, D. Ávila Brande, I. Terry, E. Morán, R. Schmidt, Microwave-assisted synthesis, microstructure, and magnetic properties of rare-earth 
cobaltites, Inorg. Chem. 56 (1) (2017) 627.

[26] M.W. Haverkort, Z. Hu, J.C. Cezar, T. Burnus, H. Hartmann, M. Reuther, C. Zobel, T. Lorenz, A. Tanaka, N.B. Brookes, et al., Spin state transition in $\mathrm{LaCoO}_{3}$ studied using soft X-ray absorption spectroscopy and magnetic circular dichroism, Phys. Rev. Lett. 97 (17) (2006) 176405.

[27] E. Barsukov, J. Macdonald, Impedance Spectroscopy: Theory, Experiment and Applications, John Wiley \& Sons Inc, Hoboken, 2005.

[28] R. Schmidt, P.B. Lin (Ed.), Ceramic Materials Research Trends, Novascience Publishers, Hauppauge, 2007.

[29] T.B. Adams, D.C. Sinclair, A.R. West, Giant barrier layer capacitance effects in $\mathrm{CaCu}_{3} \mathrm{Ti}_{4} \mathrm{O}_{12}$ ceramics, Adv. Mater. 14 (18) (2002) 1321.

[30] R. Schmidt, M.C. Stennett, N.C. Hyatt, J. Pokorny, J. Prado-Gonjal, M. Li, D.C. Sinclair, Effects of sintering temperature on the internal barrier layer capacitor (IBLC) structure in $\mathrm{CaCu}_{3} \mathrm{Ti}_{4} \mathrm{O}_{12}$ (CCTO) ceramics, J. Eur. Ceram. Soc. 32 (12) (2012) 3313 .
[31] R. Schmidt, B. Kharisov, O. Kharissova, U. Ortiz-Mendez (Eds.), CRC Concise Encyclopedia of Nanotechnology, CRC Press Taylor \& Francis Group, Boca Raton (USA), 2015.

[32] A. Wypych, I. Bobowska, M. Tracz, A. Opasinska, S. Kadlubowski, A. KrzywaniaKaliszewska, J. Grobelny, P. Wojciechowski, Dielectric properties and characterisation of titanium dioxide obtained by different chemistry methods, J. Nanomater. 2014 (2014) 9.

[33] F.D. Morrison, D.C. Sinclair, A.R. West, Electrical and structural characteristics of lanthanum-doped barium titanate ceramics, J. Appl. Phys. 86 (11) (1999) 6355.

[34] C.A. Randall, R. Eitel, B. Jones, T.R. Shrout, D.I. Woodward, I.M. Reaney, Investigation of a high Tc piezoelectric system: $(1-\mathrm{x}) \mathrm{Bi}\left(\mathrm{Mg}_{1 / 2} \mathrm{Ti}_{1 / 2}\right) \mathrm{O}_{3}-(\mathrm{x}) \mathrm{PbTiO}_{3}$, J. Appl. Phys. 95 (7) (2004) 3633.

[35] J.T.S. Irvine, D.C. Sinclair, A.R. West, Electroceramics: characterization by impedance spectroscopy, Adv. Mater. 2 (3) (1990) 132. 